\title{
Inhaltsverzeichnifs des acht und vierzigsten Bandes, nach den Gegenständen.
}

\section{Reine Mathematik.}

Abhandlung.
Nr. der

1. A n a 1 y s i s.

2. Application des transcendantes abéliennes à la théorie des fractions continues. Par Mr. C. W. Borchardt de Berlin. . . . . . . . . .

4. Werthung der Factorielle $\left(\begin{array}{c}m \\ k\end{array}\right)=\frac{m(m-1)(m-2)(m-3) \ldots(m-k+1)}{1 \cdot 2 \cdot 3 \cdot 4 \ldots \ldots k}$, beim unendlichen Zunehmen der reellen, ganzen und positiven Zahl $k$, wenn $m$ irgend eine reelle oder imaginäre Zahl ist. Von dem Herrn Dr. Raabe, Professor der Math. an der Universität zu Zürich. . . . . . .

5. Über Producte und Potenzen bestimmter einfacher Integral-Ausdrücke, durch mehrfache dargestellt. Von Demselben. (Aus den Mittheilungen der naturforschenden Gesellschaft zu Zürich.) . . . . . . . . . . . . .

6. Über die Darstellung einer Function zweier Variabeln $z, z^{\prime}$ nach aufsteigenden Potenzen zwei anderer Variabeln $y, y^{\prime}$, deren geg̣enseitige $\mathbf{A b}$ hångigkeit die Gleichungen

$$
z=x+y f(z), \quad z^{\prime}=x^{\prime}+y^{\prime} f\left(z^{\prime}\right)
$$

ausdrücken, wo $f(z)$ dieselbe Function von $z$ ist, wie $f\left(z^{\prime}\right)$ von $z^{\prime}$. Von Demselben. (Aus den Mittheilungen der naturforschenden Gesellschaft zu Zürich.)

7. Über die singulären Integral-Auflösungen einer Differentialgleichung erster Ordnung mit zwei Variabeln. Von Demselben. (Aus den Mittheilungen der naturforschenden Gesellschaft zu Zürich.) . . . . . . . . . .

8. Über einen Hülfssatz zur Ausmittelung der Werthe bestimmter Integrale. Von Demselben. (Aus den Mittheilungen der naturforschenden Gesellschaft zu Zürich.) . . . . . . . . . . . . . . . . . . . . . .

9. Zurückführung der Wurzelform einer algebraischen Gleichung auf die Integration linearer partieller, oder auch eines Systems simultaner gemeiner Differentialgleichungen erster Ordnung. Von Demselben. (Aus den Mittheilungen der naturforschenden Gesellschaft zu Zürich.) . . . . . .

10. Uber den gegenseitigen Zusammenhang einiger Functionen. Von Demselben. (Aus den Mittheilungen der naturforschenden Gesellschaft zu Zürich.). .

11. Independente Berechnung der Sturmschen Reste. Von Herrn Dr. phil. Heilermann zu Trier. . . . . . . . . . . . . . . . . . .

12. Die einfachsten periodischen Functionen. Von dem Herrn Professor Dr. Schellbach zu Berlin. . . . . . . . . . . . . . . . . . .

13. Untersuchungen über ganze Functionen. Von Herrn Prof. Dr. Heine zu Bonn.

14. Fernere Untersuchungen über ganze Functionen. Von Demselben. . . . III 243
I. 69

II. 130

II. 137 
15. Über die Entwickelung von Wurzeln algebraischer Gleichungen in Potenzreihen. Von Herrn Prof. Dr. Heine zu Bonn.

17. Über eine Function von drei Winkeln, deren erste Abgeleiteten ebenfalls als Winkel anzusehen und durch algebraische Relationen ihrer Cosinus zu denen der Unabhängigen bestimmt sind. Von Herrn Dr. Schläffli, Docenten an der Universität zu Bern. . . . . . . . . . . . . . . .

III. 292

18. Observationes quaedam in theoria numerorum. Auct. Dr. E. Meifsel, Berol.

IV. 301

20. Beitrag zur Theorie der $\boldsymbol{n}$ fach unendlichen $\Theta$ Reihen. Von Demselben. . IV. 324

21. Multiplications-Formeln für die elliptischen Functionen mit complexen Vielfachen des Arguments und dem Modul $\sqrt{\frac{1}{2}}$. Von Herrn Dr. H. Hoffmanı in Danzig.

IV. 332

24. Bemerkung zur Theorie des Gröfsten und Kleinsten. Von Herrn Sïmon Spitzer, Assistent und Privatdocent der Mathematik am K. K. polytechnischen Institute zu Wien.

25. Bemerkungen üher den Sturmschen Satz. Von Herrn Prof. F. Joachimsthal zu Halle a. d. S.

$$
\text { 2. G e o m e t r i e. }
$$

3. Über die gegenseiligen Beziehungen zwischen irgend einer ebenen Curve und den ihr zugehörigen, einem gemeinschaftlichen Pole entsprechenden Directrix - und Fufspunctenlinien. Von dem Herrn Dr. Ruabe, Professor der Math. an der Universität zu Zürich. . . . . . . . . . . . .

17. Über eine Function von drei Winkeln, deren erste Abgeleiteten ebenfalls als Winkel anzusehen und durch algebraische Relationen ihrer Cosinus zu denen der Unabhängigen bestimmt sind. Von Herrn Dr. Schläffli, Docenten an der Universität zu Bern.

23. Sur la construction des normales qu'on peut abaisser d'un point donné sur une section conique complètement décrite. Par M. F. Joachimsthal, prof. de math. à l'Université de Halle.

3. M e h a n i k.

1. Mémoire sur la question réciproque du centre de percussion. Par M. Steichen, professeur à l'école militaire de Bruxelles.

19. Zur Theorie der Taulochronen. Von Herrn Dr. phil. Meifsel zu Berlin.

IV. 317

\section{Angewand te Mathematik.}

1. Mémoire sur la question réciproque du centre de percussion. Par $M$. Steichen, professeur à l'école militaire de Bruxelles. . . . . . ..$~$
Über die Zapfenreibung bei stehenden Wellen. Von Herrn Dr. Druckenmïller, Director des Königl. Gewerbe-Instituls zu Berlin. . . . . . .

22. Über den inducirlen Magnetismus eines unbegrenzlen Cylinders von weichem Eisen. Von Herrn (i. Kirchhoff, Professor an der Universität zu Breslau. IV. 348 